hep-ph/0203237

\title{
Minimal Schemes for Large Neutrino Mixings with Inverted Hierarchy
}

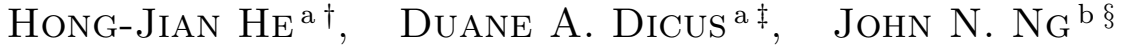 \\ ${ }^{a}$ Center for Particle Physics, University of Texas at Austin, Austin, Texas 78712, USA \\ b TRIUMF, 4004 Wesbrook Mall, Vancouver, BC V6T 2A3, Canada
}

\begin{abstract}
Existing oscillation data point to nonzero neutrino masses with large mixings. We analyze the generic features of the neutrino Majorana mass matrix with inverted hierarchy and construct realistic minimal schemes for the neutrino mass matrix that can explain the large (but not maximal) $\nu_{e}-\nu_{\mu}$ mixing of MSW-LAM as well as the nearly maximal $\nu_{\mu}-\nu_{\tau}$ mixing and the small (or negligible) $\nu_{e} \rightarrow \nu_{\tau}$ transition. These minimal schemes are quite unique and turn out to be extremely predictive. Implications for neutrinoless double beta decay, tritium beta decay and cosmology are analyzed.
\end{abstract}

PACS numbers: 14.60.Pq, 12.15.Ff, 13.15.+g, 13.40.Em

[March, 2002]

\footnotetext{
${ }^{\dagger}$ Electronic address: hjhe@physics.utexas.edu

¥ Electronic address: phbd057@utxvms.cc.utexas.edu

$\S$ Electronic address: misery@triumf.ca
} 


\section{Introduction}

The large, rather than small, neutrino mixings confirmed by atmospheric and solar oscillation experiments [1, 2] over the recent years have brought neutrino physics to an exciting new era. It indicates that lepton flavor mixing is very different from quark flavor mixing, and neutrino mass generation may have a distinct origin from the traditional Dirac-type Yukawa interactions for the charged quarks and leptons in the standard model (SM). In fact, the neutrino masses can be naturally of Majorana nature, generated from either a seesaw mechanism [3] at high scales or a radiative mechanism around the weak scale [4, 河].

The current global fit strongly favors Mikheyev-Smirnov-Wolfenstein Large Angle Mixing (MSWLAM) in which the solar mixing angle $\theta_{\odot}$ (giving the $\nu_{e} \leftrightarrow \nu_{\mu}$ transition) is large but significantly deviates from the maximal value $45^{\circ}$, i.e., $25^{\circ} \leq \theta_{\odot} \leq 39^{\circ}$ at $95 \%$ C.L.円, with a central value at $\theta_{\odot} \simeq 32^{\circ}$ [6, 8, 8]. On the other hand, the atmospheric data indicate a maximal mixing angle $\theta_{\text {atm }}$ (representing the $\nu_{\mu} \rightarrow \nu_{\tau}$ transition), with the $95 \%$ C.L. limit $33^{\circ} \leq \theta_{\text {atm }} \leq 57^{\circ}$ and the central value $\theta_{\mathrm{atm}} \simeq 45^{\circ}$ [1]. This is also supported by the K2K long baseline experiment [9]. The Chooz 10] and Palo Verde [11] long baseline reactor experiments (in combination with the mass range of atmospheric data [1]) bound $\sin ^{2} \theta_{\mathrm{chz}} \lesssim 0.04$ at $95 \%$ C.L., where the angle $\theta_{\operatorname{chz}}$ measures the $\nu_{e} \rightarrow \nu_{\tau}$ transition. Furthermore, the solar oscillations constrain the mass-square difference $\Delta_{\odot}=\left|m_{1}^{2}-m_{2}^{2}\right|$ to be, $1.8 \times 10^{-5} \mathrm{eV}^{2} \leq \Delta_{\odot} \leq 4.1 \times 10^{-4} \mathrm{eV}^{2}$, for MSW-LAM at $99 \%$ C.L., while the atmospheric oscillations confine the mass-square difference $\Delta_{\text {atm }}=\left|m_{1,2}^{2}-m_{3}^{2}\right|$ as, $1.3 \times 10^{-3} \mathrm{eV}^{2} \leq \Delta_{\text {atm }} \leq 5.5 \times 10^{-3} \mathrm{eV}^{2}$, at $99 \%$ C.L. This suggests two generic patterns for the light neutrino mass-eigenvalues, $\left(m_{1}, m_{2}, m_{3}\right) \geq 0$, namely, the "Normal Hierarchy" (called Type-A) and "Inverted Hierarchy" (called Type-B),

$$
\text { A: } m_{1}<m_{2} \ll m_{3} ; \quad \text { B: } m_{1} \sim m_{2} \gg m_{3} \text {. }
$$

Hereafter, we will focus on the Type-B scenario with inverted mass hierarchy. Our present goal is to construct a realistic scheme for the neutrino Majorana mass matrix, containing only a minimal set of parameters to describe the neutrino data, especially the non-maximal solar neutrino mixing à la MSW-LAM (which is hard [7, 12] to realize in models with an approximate $L_{e}-L_{\mu}-L_{\tau}$ symmetry [13]). We show that such a Minimal Scheme can be quite uniquely derived and is highly predictive. Implications for neutrinoless double $\beta$ decay, tritium $\beta$ decay, and cosmology are analyzed.

\section{Minimal Schemes for Neutrino Majorana Masses with Inverted Hierarchy}

Consider the generic $3 \times 3$ symmetric Majorana mass matrix $M_{\nu}$ for 3 light flavor-neutrinos $\left(\nu_{e}, \nu_{\mu}, \nu_{\tau}\right)$, at the weak scale and with leptons in the mass-eigenbasis,

$$
M_{\nu}=\left(\begin{array}{ccc}
m_{e e} & m_{e \mu} & m_{e \tau} \\
m_{e \mu} & m_{\mu \mu} & m_{\mu \tau} \\
m_{e \tau} & m_{\mu \tau} & m_{\tau \tau}
\end{array}\right) .
$$

With extra new heavy fields integrated out, Eq. (2) is the most general description of the Majorana masses of three active neutrinos based upon Weinberg's unique dimension-5 effective operator [14], $\frac{\mathcal{C}_{i j}}{\Lambda} L_{i}^{\alpha} L_{j}^{\beta} H^{\alpha^{\prime}} H^{\beta^{\prime}} \epsilon^{\alpha \alpha^{\prime}} \epsilon^{\beta \beta^{\prime}}$, which gives a mass term, $\frac{1}{2} \nu^{T} M_{\nu} \nu$, with $M_{\nu}^{i j}=\mathcal{C}_{i j} v^{2} / \Lambda$, where $\langle H\rangle=$

\footnotetext{
${ }^{1}$ The maximal value $45^{\circ}$ is also excluded by the $99 \%$ C.L. limit of the MSW-LAM solution, $24^{\circ} \leq \theta_{\odot} \leq 43^{\circ}$, [6, 再]
} 
$v / \sqrt{2}$ is the vacuum expectation value of the SM Higgs doublet. The mass matrix (2) contains nine independent real parameters, which can be equivalently chosen as three mass eigenvalues $\left(m_{1}, m_{2}, m_{3}\right) \geq 0$, three mixing angles $\left(\theta_{12}, \theta_{23}, \theta_{13}\right)$, and three CP-violation phases $\left(\phi, \phi^{\prime}, \phi^{\prime \prime}\right)$ with $\phi$ the usual Dirac phase and $\left(\phi^{\prime}, \phi^{\prime \prime}\right)$ the Majorana phases (which do not affect the neutrino oscillation). The neutrino mixing matrix $V \equiv U U^{\prime}$ for diagonalizing $M_{\nu}$, via $V^{T} M_{\nu} V=M_{\nu}^{\text {diag }}$, contains six parameters (three rotation angles and three phases) and can be decomposed into a matrix $U$ (à la Cabibbo-Kobayashi-Maskawa) and a diagonal matrix $U^{\prime}$ with only two Majorana phases,

$$
U=\left(\begin{array}{ccc}
c_{1} c_{3} & -s_{1} c_{3} & -s_{3} e^{-i \phi} \\
s_{1} c_{2}-c_{1} s_{2} s_{3} e^{i \phi} & c_{1} c_{2}+s_{1} s_{2} s_{3} e^{i \phi} & -s_{2} c_{3} \\
s_{1} s_{2}+c_{1} c_{2} s_{3} e^{i \phi} & c_{1} s_{2}-s_{1} c_{2} s_{3} e^{i \phi} & c_{2} c_{3}
\end{array}\right)
$$

and $U^{\prime}=\operatorname{diag}\left(1, e^{i \phi^{\prime}}, e^{i \phi^{\prime \prime}}\right)$. Here we use the notations $\left(\theta_{1}, \theta_{2}, \theta_{3}\right) \equiv\left(\theta_{12}, \theta_{23}, \theta_{13}\right)$, for convenience. From the mass diagonalization, we can reconstruct the neutrino mass matrix $M_{\nu}$ via the relation,

$$
M_{\nu}=V^{*} M_{\nu}^{\operatorname{diag}} V^{\dagger}
$$

which gives,

$$
\begin{aligned}
& m_{e e}=c_{3}^{2}\left[c_{1}^{2} m_{1}+s_{1}^{2} m_{2}^{\prime}\right]+p^{2} s_{3}^{2} m_{3}^{\prime}, \\
& m_{\mu \mu}=\left(s_{1} c_{2}-\bar{p} c_{1} s_{2} s_{3}\right)^{2} m_{1}+\left(c_{1} c_{2}+\bar{p} s_{1} s_{2} s_{3}\right)^{2} m_{2}^{\prime}+s_{2}^{2} c_{3}^{2} m_{3}^{\prime}, \\
& m_{\tau \tau}=\left(s_{1} s_{2}+\bar{p} c_{1} c_{2} s_{3}\right)^{2} m_{1}+\left(c_{1} s_{2}-\bar{p} s_{1} c_{2} s_{3}\right)^{2} m_{2}^{\prime}+c_{2}^{2} c_{3}^{2} m_{3}^{\prime}, \\
& m_{e \mu}=c_{3}\left[s_{1} c_{1} c_{2}\left(m_{1}-m_{2}^{\prime}\right)-\bar{p} s_{2} s_{3}\left(c_{1}^{2} m_{1}+s_{1}^{2} m_{2}^{\prime}\right)+p s_{2} s_{3} m_{3}^{\prime}\right], \\
& m_{e \tau}=c_{3}\left[s_{1} c_{1} s_{2}\left(m_{1}-m_{2}^{\prime}\right)+\bar{p} c_{2} s_{3}\left(c_{1}^{2} m_{1}+s_{1}^{2} m_{2}^{\prime}\right)-p c_{2} s_{3} m_{3}^{\prime}\right], \\
& m_{\mu \tau}=\left(s_{1} s_{2}+\bar{p} c_{1} c_{2} s_{3}\right)\left(s_{1} c_{2}-\bar{p} c_{1} s_{2} s_{3}\right) m_{1}+\left(c_{1} s_{2}-\bar{p} s_{1} c_{2} s_{3}\right)\left(c_{1} c_{2}+\bar{p} s_{1} s_{2} s_{3}\right) m_{2}^{\prime}-s_{2} c_{2} c_{3}^{2} m_{3}^{\prime},
\end{aligned}
$$

where $\left(m_{2}^{\prime}, m_{3}^{\prime}\right) \equiv\left(m_{2} e^{-i 2 \phi^{\prime}}, m_{3} e^{-i 2 \phi^{\prime \prime}}\right)$ and $p=\bar{p}^{*} \equiv e^{i \phi}$.

The precise form of the neutrino mass matrix $M_{\nu}$ in Eq. (2) should be predicted by an appropriate full theory where the mass-mechanism is known. On the other hand, Eq. (4) shows how $M_{\nu}$ can be fully reconstructed in terms of nine directly measurable quantities, the mass-eigenvalues, the mixing angles and the CP-phases. Before knowing the underlying full theory, this suggests an important and reliable bottom-up approach, namely, we ask: given the existing neutrino experiments, can we construct a simple, realistic $M_{\nu}$ with only a minimal set of input parameters which describes all the oscillation data? To be concrete, we will focus on the Type-B scenario with inverted mass hierarchy in Eq. (1).2 We will show that such a minimal scheme can be quite uniquely derived and is highly predictive. We can, of course, further extend or elaborate the Minimal Scheme with more fine structure and more input parameters if that is needed to match with an underlying theory (once specified). However, the essential structure of the Minimal Scheme and its capability for describing

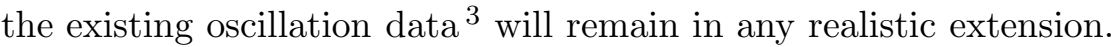

\subsection{Minimal Scheme of Type-B1}

The neutrino mass matrices of inverted hierarchy (Type-B) can be classified into Type-B1 and -B2 [17] which we will analyze in turn. We start from the simplest, naive mass matrix $M_{\nu 0}$ of

\footnotetext{
${ }^{2}$ For a very recent analysis of the normal hierarchy (Type-A) via a bottom-up approach, see Ref. 15.

${ }^{3}$ The result from Liquid Scintillation Neutrino Detector (LSND) 16 awaits confirmation by the Fermilab miniBooNE experiment [18] and will not be considered in the present study.
} 
Type-B1 [17],

$$
M_{\nu 0}[\mathrm{~B} 1]=\frac{\bar{m}_{0}}{\sqrt{2}}\left(\begin{array}{lll}
0 & 1 & 1 \\
1 & 0 & 0 \\
1 & 0 & 0
\end{array}\right),
$$

which generates a mass spectrum $\left(m_{1}, m_{2}, m_{3}\right)=\left(m_{1},-m_{2}^{\prime}, m_{3}^{\prime}\right)=\bar{m}_{0}(1,1,0)$ and exact bimaximal mixing, $\theta_{1}=\theta_{2}=45^{\circ}$. This simple structure (6) is motivated by $L_{e}-L_{\mu}-L_{\tau}$ symmetry円. (It was also shown to be generic for the minimal radiative Zee-model and its various extensions [4, 5, 20].) However, (6) is not realistic and is excluded by the solar oscillation data since it predicts $\Delta_{\odot}=\left|m_{1}^{2}-m_{2}^{2}\right|=0$, and, more seriously, a maximal solar angle $\theta_{\odot}=\theta_{1}=45^{\circ}$ which is difficult to reconcile with the MSW-LAM 12] [7]. We observe that such a failure is due to the small but nonzero ratio of the two measured mass-square differences $\Delta_{\odot} / \Delta_{\text {atm }}=\mathcal{O}\left(10^{-1}-10^{-2}\right)$ and a moderate angular deviation $\left(45^{\circ}-\theta_{1}\right) / \theta_{1} \sim\left(45^{\circ}-32^{\circ}\right) / 32^{\circ} \sim 0.4$, for MSW-LAM. Therefore, it is justified to take $M_{\nu 0}$ as our zeroth order mass matrix and build in the necessary Minimal Perturbations to make a realistic Type-B1 neutrino mass-matrix $M_{\nu}=M_{\nu 0}+\Delta M_{\nu}$. Such minimal perturbations represent proper $L_{e}-L_{\mu}-L_{\tau}$ violation effects. What is the minimal set of extra parameters which we need for a realistic perturbation $\Delta M_{\nu}$ ? First, we need a sizable parameter $\kappa=\mathcal{O}(0.5)$ to accommodate the solar angular deviation of $\left(45^{\circ}-\theta_{1}\right) / \theta_{1} \sim 0.4$; second, we need a small parameter $\delta^{\prime} \sim\left|m_{1}-m_{2}\right| / \bar{m}_{0} \lesssim \mathcal{O}(0.1)$ to account for the minor mass ratio $\Delta_{\odot} / \Delta_{\text {atm }}=\mathcal{O}\left(10^{-1}-10^{-2}\right)$; finally, to ensure the Type-B mass spectrum (1) we should impose a condition $\left|m_{1}-m_{2}\right| / \bar{m}_{0} \sim$ $m_{3} / \bar{m}_{0}=\mathcal{O}\left(\delta^{\prime}\right)$, which can be naturally realized only if we introduce an "interplay" parameter $\delta$ lying between $\kappa$ and $\delta^{\prime}$. In summary, to construct a realistic perturbation to $M_{\nu 0}$, we have to start with three dimensionless parameters $\left(\kappa, \delta, \delta^{\prime}\right)$ satisfying the proper hierarchies,

$$
\begin{aligned}
& 1>|\kappa|>|\delta|>\left|\delta^{\prime}\right|, \quad|\kappa| \gg\left|\delta^{\prime}\right|, \\
& m_{3} / \bar{m}_{0} \sim\left|m_{1}-m_{2}\right| / \bar{m}_{0}=\mathcal{O}\left(\delta^{\prime}\right) .
\end{aligned}
$$

With these, we can almost uniquely determine the pattern of the perturbation $\Delta M_{\nu}$, and derive the following Minimal Scheme-B1:

$$
M_{\nu}[\mathrm{B} 1]=\frac{\bar{m}_{0}}{\sqrt{2}}\left(\begin{array}{ccc}
\kappa & 1 & 1 \\
1 & -\kappa & -\delta \\
1 & -\delta & -\delta^{\prime}
\end{array}\right) .
$$

The relative sign between 11- and 22-entry is uniquely fixed by the requirement $\left|m_{1}-m_{2}\right| / \bar{m}_{0}=$ $\mathcal{O}\left(\delta^{\prime}\right)$. Note that to affect $\theta_{1}, \kappa$ cannot be put in 12- and 21-entry as $M_{\nu}$ is symmetric. Another reason to arrange the 11-entry to be of $\mathcal{O}(\kappa)$ rather than $\mathcal{O}\left(\delta, \delta^{\prime}\right)$ comes from the generic observation about the nature of $m_{e e}$ by using the Type-B mass spectrum (11) and the general Eq. (5),

$$
m_{1} c_{3}^{2} \gtrsim\left|m_{e e}\right| \gtrsim m_{1} c_{3}^{2}\left|\cos 2 \theta_{1}\right| \simeq m_{1}\left|\cos 2 \theta_{1}\right|,
$$

where the upper [lower] bound corresponds to the CP-conserving values of the Majorana phase $\phi^{\prime}=0$, or, $\pi[\pi / 2$, or, $3 \pi / 2]$. For solar mixing within the $95 \%$ C.L. range, $25^{\circ} \leq \theta_{1} \leq 39^{\circ}$ (MSW-LAM), we deduce the lower limit,

$$
\left|m_{e e}\right| / m_{1} \approx\left|m_{e e}\right| / \bar{m}_{0} \gtrsim(0.21-0.64)
$$

\footnotetext{
${ }^{4}$ For some recent non-minimal approaches with certain $U(1)$ flavor symmetry and additional fields, see Ref. 21].
} 
so that we can identify $\sqrt{2}\left|m_{e e}\right| / \bar{m}_{0}=\mathcal{O}(\kappa)$. It is important to note that, for general Type-B scenarios, the significant deviation of $0.15 \leq\left(45^{\circ}-\theta_{1}\right) / \theta_{1} \leq 0.8$ for $25^{\circ} \leq \theta_{1} \leq 39^{\circ}$ [6] à la MSWLAM already requires a sizable $m_{e e}$ which is potentially observable via $0 \nu \beta \beta$-decay experiments [22], depending on the overall scale $\bar{m}_{0}$. As will be shown in Sec. 3.1, due to the condition (7) and the smallness of $s_{3}^{2}$ [10], we can reduce the dimensionless inputs $\left(\kappa, \delta, \delta^{\prime}\right)$ down to a single parameter $\kappa$ (or, equivalently, the solar angle $\theta_{\odot} \simeq \theta_{1}$ ). This makes our minimal scheme-B1 highly predictive.

After a scan for all possible variations of the minimal Scheme-B1 under the condition (7), we find a few other acceptable minimal schemes with $M_{\nu} \equiv\left(\bar{m}_{0} / \sqrt{2}\right) \mathfrak{M}$ and $\mathfrak{M}$ given by

$$
\left(\begin{array}{ccc}
\kappa & 1 & 1 \\
1 & -\delta^{\prime} & -\delta \\
1 & -\delta & -\kappa
\end{array}\right), \quad\left(\begin{array}{ccc}
\kappa & 1-\delta^{\prime} & 1 \\
1-\delta^{\prime} & -\kappa & -\delta \\
1 & -\delta & 0
\end{array}\right), \quad\left(\begin{array}{ccc}
\kappa & 1-\delta^{\prime} & 1 \\
1-\delta^{\prime} & 0 & -\delta \\
1 & -\delta & -\kappa
\end{array}\right) .
$$

Here the first matrix has the same mass eigenvalues as Eq. (8); its rotation angles $\left(\theta_{3}, \theta_{2}\right)$ contain a sign flip for the small $\mathcal{O}\left(s_{3}\right)$ terms. The third matrix in Eq. (11) is a variation of the first matrix by relocating $\delta^{\prime}$; similarly, the second matrix above is constructed by relocating $\delta^{\prime}$ from Eq. (8). Hence, Eq. (11) differs from Eq. (8) only by small terms of $\mathcal{O}\left(s_{3}, \delta^{\prime}\right)$, with no conceptual difference. We will focus on the minimal scheme (8) hereafter. We also note that all the realistic minimal schemes we find for the Type-B can have at most one independent texture zero [cf. the above Eq. (11) and the following Eq. (13) with $\xi^{\prime}=0$ or $\xi=0$ ]. A recent interesting analysis [19] classified viable schemes with two independent texture-zeros, which, as expected, do not contain Type-B schemes.

\subsection{Minimal Scheme of Type-B2}

The naive form of Type-B2 is defined as [17,

$$
M_{\nu 0}[\mathrm{~B} 2]=m_{0}\left(\begin{array}{ccc}
1 & 0 & 0 \\
0 & 1 / 2 & 1 / 2 \\
0 & 1 / 2 & 1 / 2
\end{array}\right)
$$

which has a Type-B mass-spectrum $\left(m_{1}, m_{2}, m_{3}\right)=\left(m_{1}, m_{2}^{\prime}, m_{3}^{\prime}\right)=m_{0}(1,1,0)$, a maximal mixing angle $\theta_{2}=45^{\circ}$, and vanishing $\left(\theta_{1}, \theta_{3}\right)$. To be realistic, the zeroth order matrix (12) has to be properly perturbed for generating the observed small but nonzero $\Delta_{\odot}=\left|m_{1}^{2}-m_{2}^{2}\right|$ and the large (rather than maximal) mixing $\theta_{1}$ for MSW-LAM. Using the fact of $m_{1} \simeq m_{2} \gg m_{3}$ and the general relation (5), we find all the perturbations in $\Delta M_{\nu}=M_{\nu}-M_{\nu 0}$ to be of $\mathcal{O}\left(m_{1}-m_{2}, m_{3}, s_{3}^{2} m_{0}\right)$ or smaller. This suggests a completely different perturbation structure from Type-B1, namely, we use only small perturbation parameters of $\mathcal{O}\left(\left|m_{1}-m_{2}\right| / m_{0}, m_{3} / m_{0}\right)$ and the large solar mixing angle $25^{\circ} \leq \theta_{1} \leq 39^{\circ}$ (95\% C.L.) can be naturally generated from an $\mathcal{O}(1)$ ratio of two small perturbation parameters. Inspecting the structure of $\Delta M_{\nu}=M_{\nu}-M_{\nu 0}$ for Type-B2 and using Eq. (5), we are quite uniquely led to the following Scheme-B2,

$$
M_{\nu}[\mathrm{B} 2]=m_{0}\left(\begin{array}{ccc}
1+\delta & \xi & \xi^{\prime} \\
\xi & 1 / 2 & 1 / 2 \\
\xi^{\prime} & 1 / 2 & 1 / 2
\end{array}\right)
$$

where we impose

$$
\frac{m_{3}}{m_{0}} \lesssim \frac{\left|m_{1}-m_{2}\right|}{m_{0}}=\mathcal{O}\left(\delta, \xi, \xi^{\prime}\right) \ll 1
$$


We can define a truly Minimal Scheme-B2 by setting $\xi^{\prime}=0$. Choosing $\xi^{\prime}=\xi$ will give, $\theta_{3}=$ $\mathcal{O}\left(\delta^{2}, \xi^{2}, s_{3}^{2}, \delta s_{3}, \xi s_{3}\right) \simeq 0$, and is consistent with the CHooz bound [10]. But Type-B2 generally has negligible $s_{3}$ even for nonzero $\xi^{\prime} \neq \xi$. In Eq. (13), there is no need to perturb the $2 \times 2$ block of $\nu_{\mu}-\nu_{\tau}$ as the maximal mixing is favored by the atmospheric data [1]; also the $2 \times 2$ block of $\nu_{e}-\nu_{\mu}$ invokes two small perturbations so that an $\mathcal{O}(1)$ ratio is generated to explain the non-maximal solar mixing (MSW-LAM). Unlike the Type-B1, the scheme-B2 has larger $m_{e e}=m_{0}(1+\delta) \simeq m_{0}$ at the zeroth order and is more sensitive to the $0 \nu \beta \beta$ experiments.

\section{Analysis of the Minimal Schemes and Predictions for Neutrino Oscillation}

In this section, we systematically solve the diagonalization equations in (5) for the Minimal Scheme-B1 (8) and -B2 (13) $\left[\xi^{\prime}=0\right]$ with CP-conservation. We then study their predictions for the neutrino oscillations.

\subsection{Analyzing the Minimal Scheme of Type-B1}

The parameters $(\kappa, \delta)$ will be retained up to all orders without approximation. But, from the solar and CHOOz oscillation data, it is justified to treat the small parameters $\left(\delta^{\prime}, s_{3}\right)$ as perturbations to first power and ignore terms of $\mathcal{O}\left(\delta^{\prime 2}, s_{3}^{2}\right) \lesssim \mathcal{O}\left(10^{-2}\right)$ or smaller. As will be shown below, the expansion of $s_{3}$ also plays a key role for eliminating $\delta^{\prime}$ from inputs.

From Eq. (8), we deduce the mass-eigenvalues of $M_{\nu}$, up to $\mathcal{O}\left(\overline{\delta^{\prime}}\right)$,

$$
m_{1,2}=m_{0}\left[1 \mp \frac{1}{2}\left(1-\frac{x}{2+\omega}\right) \overline{\delta^{\prime}}\right], \quad m_{3}=m_{0} \frac{x}{2+\omega} \overline{\delta^{\prime}},
$$

where we expand $\overline{\delta^{\prime}}$ to first order and define,

$$
\begin{aligned}
& \bar{\kappa} \equiv \frac{\kappa}{\bar{\omega}}, \quad \bar{\delta} \equiv \frac{\delta}{\bar{\omega}}, \quad \overline{\delta^{\prime}} \equiv \frac{\delta^{\prime}}{\bar{\omega}}, \quad \bar{\omega} \equiv \sqrt{2+\omega}=\sqrt{\frac{2}{1-\bar{\kappa}^{2}-\bar{\delta}^{2}}}, \\
& \omega \equiv \kappa^{2}+\delta^{2}=\frac{2\left(\bar{\kappa}^{2}+\bar{\delta}^{2}\right)}{1-\left(\bar{\kappa}^{2}+\bar{\delta}^{2}\right)}, \quad m_{0} \equiv \bar{m}_{0} \sqrt{1+\frac{\omega}{2}} .
\end{aligned}
$$

The parameter $x=\mathcal{O}(1)$ in Eq. (15) will be determined by the consistency condition,

$$
\left(1-\delta^{2}\right) \kappa-2 \delta+\left(1+\kappa^{2}\right) \delta^{\prime}=x \delta^{\prime}
$$

due to the requirement $m_{3} / \bar{m}_{0}=\mathcal{O}\left(\delta^{\prime}\right)$ in Eq. (7). With the definition of Eq. (16), we can rewrite the neutrino mass matrix (8) scaled by $m_{0}$,

$$
M_{\nu}[\mathrm{B} 1]=m_{0}\left(\begin{array}{ccc}
\bar{\kappa} & \bar{\omega}^{-1} & \bar{\omega}^{-1} \\
\bar{\omega}^{-1} & -\bar{\kappa} & -\bar{\delta} \\
\bar{\omega}^{-1} & -\bar{\delta} & -\bar{\delta}^{\prime}
\end{array}\right) .
$$

From Eq. (15), we deduce

$$
\frac{\Delta_{\odot}}{\Delta_{\mathrm{atm}}}=\frac{\left|m_{1}^{2}-m_{2}^{2}\right|}{\left|m_{1,2}^{2}-m_{3}^{2}\right|} \simeq 2\left[1-\frac{x}{2+\omega}\right] \overline{\delta^{\prime}}
$$

With the mass-eigenvalues given, we can then solve for the mixing angles by substituting $M_{\nu}$ [cf. Eq. (8) or Eq. (18)] into the six diagonalization equations in (5) and expanding $\left(\delta^{\prime}, s_{3}\right)$ systematically 
to first order. At this order, we find that only five of the six equations are independent. Note that we have three dimensionless parameters $\left(\kappa, \delta, \delta^{\prime}\right)$ in $M_{\nu}$ (in which the overall scale $\bar{m}_{0}$ is irrelevant to the diagonalization) and three mixing angles $\left(\theta_{1}, \theta_{2}, \theta_{3}\right)$. Hence, from the five equations, we can solve five out of the six parameters as functions of a single dimensionless input parameter which will be chosen as the angle $\theta_{1}$ (measured in the solar oscillation). To explicitly understand this nontrivial reduction of input parameters, we first note that even though we have three dimensionless inputs $\left(\kappa, \delta, \delta^{\prime}\right)$ in (8), the condition $m_{3} / \bar{m}_{0}=\mathcal{O}\left(\delta^{\prime}\right)$ in (7) [or, (17)] relates $\kappa$ and $\delta$ at zeroth order of $\delta^{\prime}$ so that only two inputs among $\left(\kappa, \delta, \delta^{\prime}\right)$ are independent under the expansion of $\delta^{\prime}$. Then, we summarize two relevant relations derived from Eq. (5) [and Eq. (18)],

$$
\begin{aligned}
& \bar{\delta}^{2}=(1-r) \bar{\kappa} \overline{\delta^{\prime}}+\mathcal{O}\left(s_{3}^{2},{\overline{\delta^{\prime}}}^{2}\right), \\
& \bar{\kappa}=2 \bar{\delta}-(1-2 r) \overline{\delta^{\prime}}+\mathcal{O}\left(s_{3}^{2}\right),
\end{aligned}
$$

with $r \equiv \frac{x}{2+\omega}$. Now we see that the absence of $\mathcal{O}\left(s_{3}\right)$ term in Eq. (20) and the smallness of $s_{3}^{2}(\lesssim 0.04$ [10]) lead us to have three constraints [two in Eq. (20) and one in Eq. (17)] among the four parameters $\left(\bar{\kappa}, \bar{\delta}, \overline{\delta^{\prime}}, x\right)$. This feature remains if we include higher order terms via iteration. This makes our scheme end up with a single input for all mixings and thus extremely predictive. After a lengthy and careful derivation, we arrive at the following complete set of solutions of our Minimal Scheme-B1, up to $\mathcal{O}\left(\overline{\delta^{\prime}}, s_{3}\right)$,

$$
\begin{aligned}
& \bar{\kappa}=\frac{8}{9} \cos 2 \theta_{1}, \quad \bar{\delta}=\frac{1}{2} \bar{\kappa}, \quad \overline{\delta^{\prime}}=\frac{\bar{\kappa}}{4(1-r)}, \\
& \theta_{2}=\frac{\pi}{4}-\frac{3-4 r}{8(1-r)} \bar{\kappa}^{2}, \quad \theta_{3} \simeq s_{3}=\frac{3-4 r}{8(1-r)} \bar{\kappa} \sqrt{1-\bar{\kappa}^{2}},
\end{aligned}
$$

where we have,

$$
2+\omega=\frac{2}{1-\frac{5}{4} \bar{\kappa}^{2}}, \quad r=\frac{1-\frac{5}{4} \bar{\kappa}^{2}}{2\left(1-\bar{\kappa}^{2}\right)}, \quad x=\frac{1}{1-\bar{\kappa}^{2}} .
$$

Finally, inputting the solar angle $\theta_{\odot}\left(\simeq \theta_{1}\right)$, we deduce the following numerical predictions,

$$
\begin{aligned}
& 25^{\circ} \leq \theta_{1} \leq 39^{\circ}, \quad[\text { Input of MSW-LAM, 95\%C.L.] } \\
& 39.8^{\circ} \leq \theta_{2} \leq 44.5^{\circ}, \quad 0.13 \geq s_{3} \simeq \theta_{3} \geq 0.046 \\
& 0.57 \geq \bar{\kappa} \geq 0.19, \quad 0.29 \geq \bar{\delta} \geq 0.092, \quad 0.25 \geq \overline{\delta^{\prime}} \geq 0.091 ; \\
& 0.28 \geq \frac{\Delta_{\odot}}{\Delta_{\text {atm }}} \geq 0.095,
\end{aligned}
$$

and also $0.44 \leq r \leq 0.50,1.48 \geq x \geq 1.01$. The results in Eq. 23) agree well with the oscillation data [1, 2, 6, 6, 10], i.e., $33^{\circ} \leq \theta_{2} \simeq \theta_{\text {atm }} \leq 57^{\circ}$ and $s_{3}=\sin \theta_{\text {chz }} \lesssim 0.2$ at $95 \%$ C.L., and 4.2 [3.3] $\times$ $10^{-3} \leq \Delta_{\odot} / \Delta_{\text {atm }} \leq 0.17$ [0.32] at $95 \%[99 \%]$ C.L. The on-going KamLAND experiment 23 will more precisely test the MSW-LAM parameter space (though it will not be sensitive to $s_{3}$ [23]). We have checked the numerical accuracy of the above solutions by substituting Eq. (23) back into Eqs. (5) and (8) and evaluating the difference of the two sides in each equation. We find that the difference (uncertainty) is always less than $0.015(0.0009)$ for $\theta_{1}=25^{\circ}\left(39^{\circ}\right)$. Thus, our systematical expansion works well up to $\mathcal{O}\left(\overline{\delta^{\prime}}, s_{3}\right)$, as expected, since the ignored terms are of $\mathcal{O}\left({\overline{\delta^{\prime}}}^{2}, s_{3}^{2}\right)$ and 
become smaller for larger $\theta_{1}$ as shown in Eq. (23). For Type-B schemes, the mass scale $m_{0}$ is generally bounded by

$$
0.036 \mathrm{eV} \leq m_{0} \simeq m_{1,2} \simeq \Delta_{\mathrm{atm}}^{1 / 2} \leq 0.074 \mathrm{eV}, \quad[99 \% \text { C.L. }] .
$$

\subsection{Analyzing the Minimal Scheme of Type-B2}

We now turn to the minimal scheme-B2 in (13) with $\xi^{\prime}=0$, which has the mass-eigenvalues, up to $\mathcal{O}(\delta, \xi)$,

$$
m_{1,2}=m_{0}\left[1+\frac{\delta}{2} \pm \frac{1}{2} \sqrt{\delta^{2}+2 \xi^{2}}\right], \quad m_{3}=0 .
$$

Substituting (13) into (5), expanding up to $\mathcal{O}\left(\delta, \xi, s_{3}\right)$ and using (25), we derive the solutions,

$$
\begin{aligned}
& \theta_{1}=\frac{\pi}{4}-\frac{1}{2} \arcsin \frac{\delta}{\sqrt{\delta^{2}+2 \xi^{2}}}, \quad \theta_{2}=\frac{\pi}{4}, \quad \theta_{3}=-\frac{\xi}{\sqrt{2}}, \quad(\delta, \xi)>0 ; \\
& \text { and } \frac{\Delta_{\odot}}{\Delta_{\mathrm{atm}}}=\frac{\left|m_{1}^{2}-m_{2}^{2}\right|}{\left|m_{1,2}^{2}-m_{3}^{2}\right|}=2 \sqrt{\delta^{2}+2 \xi^{2}} .
\end{aligned}
$$

We see that $\theta_{2}$ is maximal at this order. The sizable deviation of $\theta_{1}-\frac{\pi}{4}$ is indeed naturally generated by an $\mathcal{O}(1)$ ratio of two small parameters $(\delta, \xi) \ll 1$. [Allowing $\xi^{\prime} \neq 0$, the corresponding formulas for Eqs. (25)-(26) can be directly obtained by the simple replacements, $\xi \rightarrow \xi-\xi^{\prime}$ for $\theta_{3}$ and $\xi \rightarrow \xi+\xi^{\prime}$ for all other quantities.] Using the inputs for LAM [6, 7, 8], $25^{\circ} \leq \theta_{\odot} \simeq \theta_{1} \leq 39^{\circ}$ and $4.2 \times 10^{-3} \leq \Delta_{\odot} / \Delta_{\text {atm }} \leq 0.17$ at $95 \%$ C.L., we deduce,

$$
1.2 \geq \delta / \xi \geq 0.3, \quad 1.1 \times 10^{-3} \leq \xi \leq 0.06 .
$$

Thus we have

$$
8 \times 10^{-4} \leq-\theta_{3} \leq 0.04 .
$$

The mass scale $m_{0} \simeq \Delta_{\mathrm{atm}}^{1 / 2}$ is bounded as in Eq. (24). As mentioned above, allowing nonzero $\xi^{\prime}=\mathcal{O}(\xi)$, we can derive,

$$
s_{3} \simeq \theta_{3}=\frac{\xi^{\prime}-\xi}{\sqrt{2}}
$$

which remains of the same order. Hence, $\left|\theta_{3}\right| \lesssim \mathcal{O}\left(10^{-2}\right)$ generally holds for Type-B2, implying negligible CP-violation from the Dirac phase $\phi$. It has been shown 24 that combining the data from two near-future long baseline accelerator experiments [25], the Main Injector Neutrino Oscillation Search (MINOS) and the Imaging Cosmic And Rare Underground Signals (ICARUS), may place a $95 \%$ C.L. lower bound, $\theta_{3} \geq \mathcal{O}(0.05)$ (when $\theta_{3}$ lies within their combined sensitivity), which could possibly discriminate Type-B2 from Type-B1.

\section{Implications for Neutrinoless Double $\beta$-Decay, Tritium $\beta$-Decay and Cosmology}

The oscillation data may already give a strong hint on the neutrino mass scale [cf. Eq. (24)] so long as the neutrino masses exhibit the hierarchy structure [cf. Eq. (11)], but the possibility of three nearly degenerate neutrinos $\left(m_{1} \sim m_{2} \sim m_{3}\right)$ could allow a higher scale. Hence, the laboratory experiment on neutrinoless double $\beta$-decay $(0 \nu \beta \beta)$ [22, 26, 28] is indispensable to pin 
down the absolute mass scale, as well as the Majorana nature of active neutrinos. For the Minimal Scheme-B1 and -B2, we have,

$$
m_{e e}[\mathrm{~B} 1]=\bar{\kappa} m_{0}, \quad m_{e e}[\mathrm{~B} 2] \simeq m_{0} .
$$

Thus, using Eqs. (30) and (24), we derive, at 99\% C.L.,

$$
0.014 \mathrm{eV} \leq m_{e e}[\mathrm{~B} 1] \leq 0.029 \mathrm{eV}, \quad 0.036 \mathrm{eV} \leq m_{e e}[\mathrm{~B} 2] \leq 0.074 \mathrm{eV},
$$

where we input the central value of solar fit (LAM), $\theta_{1} \simeq 32^{\circ}$, for Type-B1. The $m_{e e}[\mathrm{~B} 2]$ is already sensitive to the current $0 \nu \beta \beta$ measurement [27]. The experiments of $0 \nu \beta \beta$ decay [22], such as the on-going NEMO3 and the upcoming CuORE, can probe $\left|m_{e e}\right| \sim 0.1 \mathrm{eV}$, while the near-future measurements at Genius, Exo, Majorana and Moon aim at a sensitivity of $\left|m_{e e}\right| \sim 0.01 \mathrm{eV}$, which is decisive for testing the whole mass range (31) of Type-B1 and -B2 schemes.

Tritium $\beta$-decay requires [29], $m_{\nu_{e}}<2.2 \mathrm{eV}$, at 95\% C.L., where $m_{\nu_{e}} \equiv\left(M_{\nu}^{\dagger} M_{\nu}\right)_{e e}^{1 / 2} \simeq m_{1,2} \simeq$ $m_{0}$ for Type-B. This is well above the range given in Eq. (24). The sensitivity of $H^{3} \beta$-decay could eventually reach $m_{\nu_{e}} \sim 0.5 \mathrm{eV}$ [30].

The latest cosmology measurements of the power spectrum for the Cosmic Microwave Background (CMB), Galaxy Clustering and Lyman Alpha Forest [31] put a 95\% C.L. upper bound on the neutrino masses, $\sum_{j} m_{j} \leq 4.2 \mathrm{eV}$. This gives, for our Type-B schemes, $m_{1} \simeq m_{2} \simeq m_{0} \leq 2.1 \mathrm{eV}$, which is about the same as the tritium $\beta$-decay bound. The newest analysis [32] from the $2 \mathrm{dF}$ Galaxy Redshift Survey arrives at an upper bound, $\sum_{j} m_{j} \leq 2.2 \mathrm{eV}$, which results in, $m_{1} \simeq m_{2} \simeq$ $m_{0} \leq 1.1 \mathrm{eV}$, for Type-B schemes. Stronger constraints of $\sum_{j} m_{j} \lesssim 0.4 \mathrm{eV}$ are expected from the forthcoming Microwave Anisotropy Probe (MAP) and PLANCK satellite experiments [33, 34].

It is interesting to note that the neutrino mass scale may also be determined from the so-called $Z$-bursts [35] due to the resonant annihilation of ultra high energy neutrinos with cosmological relic (anti-)neutrinos into $Z$ bosons (whose decay produces protons and photons). In the most plausible case where the ordinary cosmic rays are protons of extragalactic origin, the required neutrino mass range is [36],

$$
0.01[0.02] \mathrm{eV} \leq m_{\nu} \text { (heaviest) } \leq 3.0[2.1] \mathrm{eV}, \quad \text { at } 99 \% \text { C.L. }[95 \% \text { C.L }],
$$

which is compatible with the neutrino oscillation bound (24) for the inverted mass hierarchy.

\section{Conclusions}

In this study, we have considered two essential and distinct scenarios for the neutrino Majorana mass matrix with inverted hierarchy, called Type-B1 [cf. Eq. (6)] and -B2 [cf. Eq. (12)]. For TypeB1, we start with the form of Eq. (6) at zeroth order and perturb it into the realistic form of Eq. (8) with three parmeters $\left(\kappa, \delta, \delta^{\prime}\right)$ under the hierarchy (7) that is necessary to correctly predict the oscillation data, especially, the non-maximal solar neutrino mixing of MSW-LAM. The sizes of $\delta^{\prime} \sim\left|m_{1}-m_{2}\right| / m_{0} \sim \Delta \odot / \Delta_{\text {atm }} \ll 1$ [2] and $s_{3}=\sin \theta_{\text {chz }} \ll 1$ [10] justify the expansion of $\left(\delta^{\prime}, s_{3}\right)$, which enables us to reduce the number of inputs down to a single parameter $\kappa$, or, equivalently, $\theta_{\odot}\left(\simeq \theta_{1}\right)$. Thus, using only the measured solar angle $\theta_{\odot}$ as input, we predict the atmospheric mixing angle, $\theta_{\mathrm{atm}}\left(\simeq \theta_{2}\right)$, the value of $\theta_{\mathrm{chz}}\left(=\theta_{3}\right)$, and the mass ratio $\Delta_{\odot} / \Delta_{\mathrm{atm}}$, in complete agreement with the existing data. We also note that the Minimal Scheme-B1 in Eq. (8) points to a generic way for 
naturally extending the minimal Zee-model [4] in which $M_{\nu}$ exhibits the following structure,

$$
M_{\nu}^{\text {Zee }}=\left(\begin{array}{ccc}
0 & m_{e \mu} & m_{e \tau} \\
m_{e \mu} & 0 & m_{\mu \tau} \\
m_{e \tau} & m_{\mu \tau} & 0
\end{array}\right),
$$

where the pattern $m_{e \mu} \simeq m_{e \tau} \gg m_{\mu \tau}$ can be realized [20], which ensures an approximate $L_{e}-L_{\mu}-$ $L_{\tau}$ symmetry. The necessity of modifying Eq. (33) in the minimal Zee-model for accommodating the MSW-LAM was noted recently [12]. Our minimal construction of Scheme-B1 in Eq. (8) demonstrates a generic way to extend $M_{\nu}^{\text {Zee }}$ under an appropriate perturbation [cf. Eq. (7)]. The choice of $m_{e e}=\left(\bar{m}_{0} / \sqrt{2}\right) \kappa=m_{0} \bar{\kappa}$ is due to the general observation in Eq. (9) for Type-B1 and a sizable $\cos 2 \theta_{1} \in(0.21-0.64)$ for $25^{\circ} \leq \theta_{1} \leq 39^{\circ}$ (95\% C.L., MSW-LAM); while $m_{\mu \mu} \simeq-m_{e e}$ is enforced by the Type-B mass-spectrum, $m_{1,2} \gg\left|m_{1}-m_{2}\right| \sim m_{3}$. The $\kappa$ terms in $M_{\nu}[\mathrm{B} 1]$ represent the generic leading modification to the minimal Zee-model (33).

For Type-B2, we start with the leading order mass matrix (12) and find the perturbation structure in Eq. (13) based on the general relations in Eq. (5) and the smalless of $\theta_{\mathrm{chz}}\left(=\theta_{3}\right)$. The realistic Minimal Scheme-B2 contains only two small parameters $(\delta, \xi) \ll 1$, defined as in Eq. (13), when $\xi^{\prime}=0$. In contrast to Typy-B1, the non-maximal solar mixing angle $\theta_{\odot}$ is naturally accommodated by a ratio of two small parameters, $\delta / \xi=\mathcal{O}(1)$, while the atmospheric mixing angle remains maximal. Using the measured values of solar angle $\theta_{\odot}$ and mass ratio $\Delta_{\odot} / \Delta_{\text {atm }}$, we derive the ranges for $\theta_{3}\left(=\theta_{\mathrm{chz}}\right)$ and the perturbation parameters $(\delta, \xi)$. The angle $\theta_{3}$ is found to be of $\mathcal{O}\left(10^{-2}\right)$ or smaller. Combining the data from both MINOS and ICARUS experiments 25] may reach the sensitivity [24] to discriminate between the minimal Type-B1 and Type-B2 schemes.

The overall neutrino mass scale $m_{0}$ for the inverted mass hierarchy is quite uniquely fixed by the atmospheric neutrino data on the mass-squared difference $\Delta_{\mathrm{atm}}=\left|m_{1,2}^{2}-m_{3}^{2}\right| \simeq m_{1,2}^{2} \simeq m_{0}^{2}$ [cf. Eq. (24)]. Thus, the mass matrix of our minimal scheme-B1 or -B2 is known and highly predictive.

Some implications of the Type-B1 and -B2 minimal schemes for the neutrinoless double $\beta$-decay, tritium $\beta$-decay and cosmology are given above.

Note Added: After the submission of this work, a new announcement [37] from the Sudbury Neutrino Observatory (SNO) collaboration appeared on April 20, 2002, which further confirms the MSW-LAM as the best solution to the solar neutrino oscillations.

\section{Acknowledgments}

We are happy to thank Vernon Barger, Danny Marfatia and Alexei Yu. Smirnov for valuable discussions. This work was supported in part by U.S. Department of Energy under grant DE-FG0393ER40757 and by Natural Science and Engineering Research Council of Canada.

\section{References}

[1] S. Fukuda, et al., [Super-Kamiokande Collaboration], Phys. Rev. Lett. 85, 3999 (2000); 86, 5656 (2001); 82, 1810 (1999); 81, 1562 (1998); T. Toshito, hep-ex/0105023.

[2] S. Fukuda, et al., [Super-Kamiokande Collaboration], Phys. Rev. Lett. 86, 5656 (2001); Q. R. Ahmad, et al., [SNO collaboration], Phys. Rev. Lett. 87, 071301 (2001). 
[3] M. Gell-Mann, P. Ramond, R. Slansky, in proceedings of the Workshop on Supergravity, p.315, 1979; T. Yanagida, proceedings of the Workshop on Unified Theories and Baryon Number in the Universe, p.79, 1979; R. N. Mohapatra, G. Senjanovic, Phys. Rev. Lett. 44, 912 (1980).

[4] A. Zee, Phys. Lett. B93, 389 (1980); Phys. Lett. B161, 141 (1985).

[5] L. Wolfenstein, Nucl. Phys. B175, 92 (1980); S. T. Petcov, Phys. Lett. B115, 401 (1982).

[6] P. I. Krastev and A. Yu. Smirnov, hep-ph/0108177; J. N. Bahcall, M. C. Gonzalez-Garcia, and C. Pena-Garay, hep-ph/0111150; G. L. Fogli, E. Lisi, D. Montanino, and A. Palazzo, Phys. Rev. D64, 093007 (2001) hep-ph/0106247 and hep-ph/0203138.

[7] F. Feruglio, A. Strumia, and F. Vissani, hep-ph/0201291; A. Strumia, private communication.

[8] M. C. Gonzalez-Garcia and Y. Nir, hep-ph/0202058.

[9] S. H. Ahn, et al., [K2K Collaboration], Phys. Lett. B511, 178 (2001).

[10] M. Apollonio, et al., [Chooz Collaboration], Phys. Lett. B466, 415 (1999); B420, 397 (1998).

[11] F. Boehm et al., [Palo Verde Collaboration], Phys. Rev. Lett. 84, 3764 (2000); Phys. Rev. D 64, 112001 (2001).

[12] Y. Koide, Phys. Rev. D64, 077301 (2001), hep-ph/0201250, hep-ph/0203113; P. H. Frampton, M. C. Oh, and T. Yoshikawa, hep-ph/0110300; B. Brahmachari and S. Choubey, hep$\mathrm{ph} / 0111133$.

[13] E.g., S. T. Petcov, Phys. Lett. B110, 245 (1982); B115, 401 (1982); R. Barbieri, et al., JHEP 9812, 017 (1998) hep-ph/9807235; R. N. Mohapatra, A. Perez-Lorenzana, C. A. de S. Pires, Phys. Lett. B474, 355 (2000); W. Grimus, L. Lavoura, JHEP 0107, 045 (2001); R. N. Mohapatra, hep-ph/0107264; K. S. Babu and R. N. Mohapatra, Phys. Lett. B532, 77 (2002).

[14] S. Weinberg, Phys. Rev. Lett. 43, 1566 (1979).

[15] M. Frigerio and A. Yu. Smirnov, hep-ph/0202247.

[16] C. Athanassopoulos, et al., [LSND Collaboration], Phys. Rev. Lett. 81, 1774 (1998).

[17] E.g., G. Altarelli and F. Feruglio, Phys. Rept. 320, 295 (2000) hep-ph/9905536.

[18] A. Bazarko, et al., [MiniBooNE Collaboration], Nucl. Phys. Proc. Suppl. 91, 210 (2001).

[19] P. H. Frampton, S. L. Glashow, and D. Marfatia, hep-ph/0201008; Z. Z. Xing, hep-ph/0201151, hep-ph/0205032.

[20] E.g., C. Jarlskog, M. Matsuda, S. Skadhauge, and M. Tanimoto, Phys. Lett. B449, 240 (1999); P. H. Frampton and S. L. Glashow, Phys. Lett. B461, 95 (1999); D. Chang and A. Zee, Phys. Rev. D61, 071303 (1999).

[21] E.g., T. Ohlsson, G. Seidl, hep-ph/0203117; Q. Shafi, Z. Tavartkiladze, hep-ph/0002150.

[22] S. R. Elliott and P. Vogel, hep-ph/0202264. 
[23] K. Inoue, [KamLAND Collaboration], talk at First Sendai International Conference on Neutrino Science, March 14-16, 2002, Sendai, Japan; V. Barger, D. Marfatia, and B. P. Wood, Phys. Lett. B498, 53 (2001) hep-ph/0011251.

[24] V. Barger, et al, Phys. Rev. D65, 053016 (2002) hep-ph/0110393.

[25] S. G. Wojcicki, [MINOS Collaboration], Nucl. Phys. Proc. Suppl. 91, 216 (2001);

A. Rubbia, [ICARUS Collaboration], talk at Skandinavian Neutrino Workshop, Uppsala, Sweden, February, 2001.

[26] H. V. Klapdor-Kleingrothaus, et al., Eur. Phys. J. A12, 147 (2001).

[27] H. V. Klapdor-Kleingrothaus, et al., hep-ph/0201231; see, however, C. E. Aalseth, et al., hep$\mathrm{ph} / 0202018$.

[28] For some recent papers on $0 \nu \beta \beta$, e.g., S. Pascoli, S. T. Petcov, L. Wolfenstein, hep-ph/0110287; S. Pascoli, S. T. Petcov, hep-ph/0111203; H. V. Klapdor-Kleingrothaus, U. Sarkar, hepph/0201224; E. Ma, hep-ph/0201225; V. Barger, S. L. Glashow, D. Marfatia, K. Whisnant, hepph/0201262; F. Feruglio, A. Strumia, F. Vissani, hep-ph/0201291; Z. Z. Xing, hep-ph/0202034; T. Hambye, hep-ph/0201307; H. Minakata, H. Sugiyama, hep-ph/0202003; N. Haba, T. Suzuki, hep-ph/0202143; M. Y. Cheng, K. Cheung, hep-ph/0203051. B. Brahmachari, E. Ma, hepph/0202262. M. Fujii, K. Hamaguchi, T. Yanagida, hep-ph/0203189.

[29] J. Bonn, et al., Nucl. Phys. Proc. Suppl. 91, 273 (2001).

[30] A. Osipowicz, et al., [KATRIN Collaboration], hep-ex/0109033; M. Fink, private communication.

[31] X. Wang, M. Tegmark, and M. Zaldarriaga, astro-ph/0105091 [v4], Phys. Rev. D(2002).

[32] O. Elgaroy, et al., astro-ph/0204152.

[33] MAP Collaboration, http://map.gsfc.gov; PLANCK Collaboration, http://astro.estec.esa.nl/ SA-general/Projects/Planck.

[34] D. J. Eisenstein, et al., astro-ph/9807130; V. Barger, et al., hep-ph/0201262.

[35] D. Fargion, B. Mele and A. Salis, Astrophys. J. 517, 725 (1999); T. J. Weiler, Astropart. Phys. 11, 303 (1999).

[36] Z. Fodor, S. D. Katz, A. Ringwald, hep-ph/0203198; Phys. Rev. Lett. (2002) hep-ph/0105064; and private communications. For an independent study, see also, D. Fargion, M. Grossi, P. G. D. Sanctis Lucentini, C. D. Troia, R. V. Konoplich, hep-ph/0108050 and astro-ph/0102426.

[37] Q. R. Ahmad, et al., [SNO Collaboration], nucl-ex/0204008 and nucl-ex/0204009; J. Klein, talk at Phenomenology Symposium 2002, Madison, Wisconsin, USA, April 22-24, 2002. For updates of the global analysis of solar neutrino oscillation data, see: V. Barger, D. Marfatia, K. Whisnant, and B. P. Wood, hep-ph/0204253; P. Creninelli, G. Signorelli and A. Strumia, hepph/0102234[v3], A. Bandyopadhyay, S. Choubey, S. Goswami and D. P. Roy, hep-ph/0204286. 